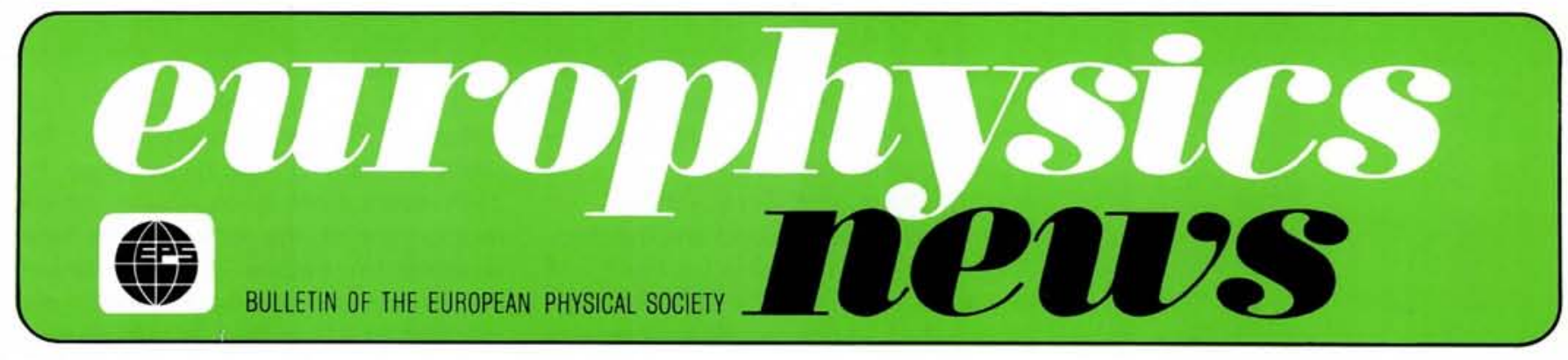

\title{
The EMC Effect
}

\section{P.B. Renton, Oxford}

(Nuclear Physics Laboratory, University of Oxford)

The scattering of high energy muons by nuclei shows an unexpected dependence upon nuclear size, suggesting that the constituent quarks and gluons may be shared between nucleons.

In our attempts to understand the elementary structure of matter, the idea of unravelling successive layers of substructure has been of central importance. Each new layer corresponds to an increasingly smaller distance scale, and it is assumed that once the physical properties of the layer in question are understood then those of the previous layer can be accounted for. This article is concerned with the subtle interplay between two layers in this hierarchy: the nucleon structure of the nucleus and the quark structure of the nucleon.

The main experimental method which has been used in probing the sub-structure of matter is the measurement of the scattering angle distribution of a beam of particles incident on the target being investigated. If the incident beam is composed of electrons, then (at currently accessible laboratory energies) the scattering is predominantly by the electromagnetic interaction. This interaction is well understood and hence the resulting scattering distributions can be converted into a measurement of the charge density distribution of the target. Historically, the idea that an atom of atomic number $Z$ is composed of a central nucleus of charge $+Z e$ (where $e$ is the charge of the proton), surrounded by $Z$ orbiting electrons each of charge $-e$, stems from the classical experiments of Rutherford. In the scattering of $\alpha$-particles (produced by radioactive decays) on thin film targets it was observed that many more $\alpha$-particles were scattered through large angles than were expected. The relative weakness of the electromagnetic coupling constant $(\alpha \cong$ $1 / 137)$, together with the large mass dif- ference between the electron and the proton or neutron, allows the large difference in the scale of the atom $\left(\cong 10^{-10}\right.$ $\mathrm{m})$ and that of the nucleus $\left(<10^{-14} \mathrm{~m}\right)$ to be understood.

Theoretically, electromagnetic interactions can be calculated, to remarkable precision, using the relativistic field theory of quantum electrodynamics (QED). Because $\alpha$ is small, a perturbative expansion in powers of $\alpha$ can be made and only the first few terms need to be calculated. Knowledge of the detailed structure of the nucleus is unimportant in accounting for most atomic properties. Turning this around, it means that little can be learnt about nuclear substructure from the study of atoms.

\section{Quark Structure of Matter}

Understanding the nucleus in terms of its components as against the atom is much more complicated. The constituent protons and neutrons (generically referred to as nucleons) have similar masses and the inter-nucleon separation ( $\cong 2 \mathrm{fm} \equiv 2 \times^{-15} \mathrm{~m}$ ) is not much

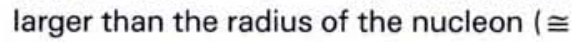
$0.8 \mathrm{fm})$. The nucleus is confined by the strong force, which can be envisaged (at least for some purposes) as being due to the exchange of pions (and other strongly interacting particles) between the nucleons. However, in the region of momentum transfers involved in nuclear binding forces, the strong interaction is not well understood. The effective strong interaction coupling constant is large in this regime, so that perturbative methods cannot be used. Thus we are left with empirical models, each with a somewhat limited range of applicability, to describe the many body gas-like system of nucleons which make up the nucleus.

The size of the nucleus (or, more strictly, of its charge distribution) and that of the proton have been determined by measuring the elastic scattering of energetic electrons by them. The mechanism for this process is shown in Fig. 1. The incident electron interacts with the target by the exchange of a photon. The photon in such an interaction is virtual (i.e. has non-zero mass). If $E$ and $P$ are the energy and momentum of the photon respectively, then its relativistically invariant mass squared is negative and is given by $E^{2}-P^{2}=-Q^{2}$, so that $Q^{2}$ is a positive quantity. The distance scale down to which the structure of the target is revealed depends on the value of $Q^{2}$. From Heisenberg's Uncertainty Principle, the distance scale resolved is roughly given by

$\Delta z \cong \hbar / \sqrt{ } Q^{2}=0.2 \mathrm{GeV} . \mathrm{fm} / \sqrt{ } Q^{2}$. (1)

\section{Contents}

The EMC Effect

Quark Gluon Plasma Research with Ultrarelativistic Heavy lons 133

Particle Accelerators in the Future

Accretion Disks and Magnetic Fields

Seminar on International Research Facilities

Nobel Prize 1988

New Members of EPS 143

Index for 1988 


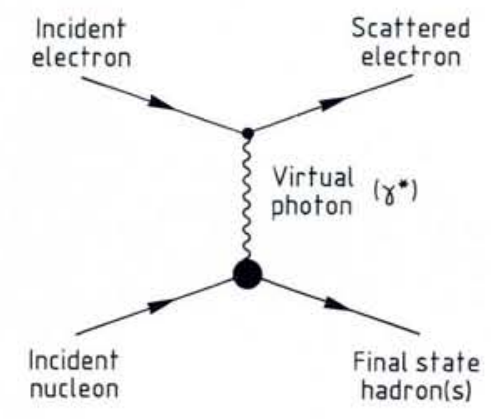

Fig. 1 - Principal mechanism in the scattering of energetic electrons from nucleons.

(In eqn. (1) $Q^{2}$ is measured in $\mathrm{GeV}^{2}$ and units in which $\hbar=c=1$ are used.) Thus, as $Q^{2}$ is increased, the structure of matter to smaller and smaller distances is revealed. The theory of QED specifies precisely the scattering distribution expected from a point-like target i.e. one with no internal structure. Deviations from these observations can be converted into a charge density distribution for the target. The dependence of the nuclear radius on the atomic weight $A$ of the target is found to behave roughly as $R=R_{0} A^{1 / 3}$, where $R_{0} \cong 1.25 \mathrm{fm}$. Thus, for a copper nucleus $(A=64), R \cong 5 \mathrm{fm}$.

The energy of the incident electron beam used to probe the size of nuclei is typically $200 \mathrm{MeV}$, giving $Q^{2}$ values of about $10^{-2} \mathrm{GeV}^{2}$. The value of $Q^{2}$ needed to probe the charge radius of a proton target is typically $\cong 0.1 \mathrm{GeV}^{2}$. This measurement is performed using the elastic scattering reaction $\mathrm{e}^{-}+\mathrm{p} \rightarrow \mathrm{e}^{-}+\mathrm{p}$, in which the same particles appear in the initial and final states.

Final states are also formed in which an excited state of the target nucleon is created (e.g. the nucleon resonance $\Delta$, which subsequently decays to a nucleon and a pion, i.e. $\Delta \rightarrow N \pi$ ). As the available energy transferred to the target is increased still further, a new phenomenon is observed. The majority of the final states is no longer elastic or quasielastic. The struck proton is observed to 'break up' and produce many strongly interacting particles (i.e. hadrons). In these inelastic final states at least one of the particles produced is either a neutron or proton (the 'baryon' quantum number is conserved), and the majority of the other relatively long-lived particles produced are pions and kaons.

What happens at the nucleon vertex (see Fig. 1) can, to a good approximation, be expressed in terms of a single, a priori unknown, function. This so-called structure function (referred to in the trade as $F_{2}$ ) is a function of the two independent kinematic variables describing the scattering process. Taking these to be $Q^{2}$ and $W$ ( $W$ is the total centre of mass energy of the final state hadrons), then the interaction probability for the incoming electron can be expressed (to a good approximation) as

$d^{2} \sigma / d Q^{2} d W=\alpha^{2}\left(K / Q^{4}\right) F_{2}\left(Q^{2}, W\right)$

where $K$ is a known kinematic function. A free photon is massless (i.e. has $E=P$ and so $Q=0$ ), and the $1 / Q^{4}$ term is a factor which expresses the suppression which arises when the photon fluctuates to a mass value of $Q$.

The construction of the two-mile long Stanford Linear Accelerator in the USA in the later 1960s extended the upper range of $Q^{2}$ which could be explored to about $10 \mathrm{GeV}^{2}$. From eqn. (1) it can be seen that this probes the proton down to distances of about $0.1 \mathrm{fm}$, much less than its radius. A study of the distributions of scattered electrons in these deep inelastic collisions was carried out.

For elastic electron-nucleon scattering the structure function $F_{2}$ was found to fall off rapidly with $Q^{2}$. A similar behaviour was also found for final states consisting of excited nucleon resonances. The experimental result that, for a fixed value of $W$ above the 'resonance region' (i.e. $W \gtrsim 2 \mathrm{GeV}$ ), the structure function $F_{2}$ was essentially independent of $Q^{2}$, was thus a great surprise. The discovery of this 'scaling' of the structure function is analogous to that of Rutherford more than 50 years earlier; only this time it showed that the proton (and neutron) had point-like constituents from which the scattering occurred. We now know that these scattering centres are the quarks which are the building blocks of hadronic matter.

A schematic diagram of the scattering process at the nucleon vertex is shown in Fig. 2. In a Lorentz frame in which the nucleon momentum is large, the motion of the quarks transverse to the virtual photon direction can be neglected. For interactions at large $Q^{2}$, the interaction time is much shorter than the typical time with which the constituents interact. Hence the cross-section is just the sum of the incoherent scatterings of the quarks. One of the nucleon's constituents, carrying a fraction $x$ of the energy-momentum four-vector $P$ of the nucleon (Fig. 2a), absorbs the virtual photon and, in some Lorentz frame, is turned around (Fig. 2b). No direct observation of these quarks has ever been made in the detectors, however, What one does observe are collimated 'jets' of hadrons; one in the direction of the scattered quark and the other in the direction of the incident target. These hadrons ( $\pi$, $K, p, \vec{p}$ etc. and also resonant states) are formed at some later time (Fig. 2c). From the relativistic kinematics of Fig. 2 , it can easily be shown that

$$
x=Q^{2} /\left(W^{2}-M_{N}^{2}+Q^{2}\right) \text {. }
$$

where $M_{N}$ is the nucleon mass. The variables $x$ and $Q^{2}$ can be measured for each event from a knowledge of the momentum of the incident and scattered electrons alone. The quark density distribution $q(x)$ and its momentum distribution $x q(x)$ can thus be built up from measurements of many such collisions.

A further surprise was in store when the results were analysed. The total momentum fraction carried by the proton's constituents (the quarks) was found to be only about 0.5 . That is, $50 \%$ of the momentum was carried by some electrically neutral constituents. We now know that these are the gluons; the particles which are responsible for the strong force confining the quarks and antiquarks inside a hadron. The gluon is analogous to the photon in QED, but whereas the photon carries no electric charge, the gluon carries the strong 'charge' (a quantum number arbitrarily called colour). This leads to an important difference between the theory of colour charges Quantum Chromodynamics or QCD) and QED. The coupling constant in QCD decreases (increases) as the separation of the quarks decreases (increases). Alternatively, in terms of the momentum transfer squared $\left(Q^{2}\right)$, the strong coupling constant $\alpha_{\mathrm{s}}$ decreases (increases) as $Q^{2}$ increases (decreases). At large $Q^{2}$, or small distances, $\alpha_{\mathrm{s}}$ becomes small and the quarks become (asymptotically) free. At small $Q^{2}$, or large distances, $\alpha_{\mathrm{s}}$ becomes large ( $(1)$, and this is believed (although not yet proved) to lead to quark confinement. The interaction mechanisms sketched in Fig. 2 can be justified in $Q C D$, at least qualitatively. For the initial virtual photon-quark collision at large $Q^{2}$, say $Q^{2}$ $\geq 10 \mathrm{GeV}^{2}, \alpha_{\mathrm{s}}$ is small ( $\cong 0.2$ ) and $\mathrm{QCD}$

(a)

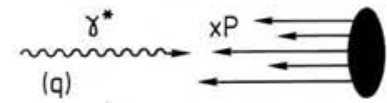
Nucleon (P)

(b)
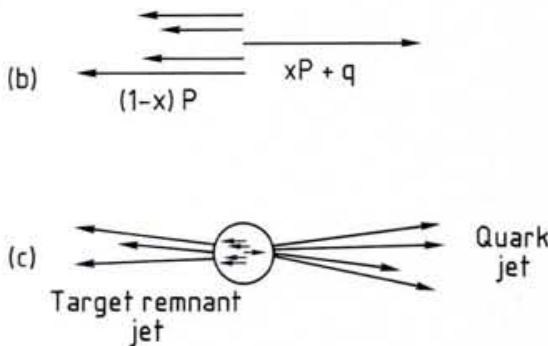

Fig. 2 - Three stages in the deep inelastic scattering process: a) just before collision b) just after collision c) some time later. The quantities, $P, x P$ and $q$ are the energy-momentum four-vectors of the incident nucleon, quark and virtual photon respectively. 
effects are relatively small. This explains why the $Q^{2}$ dependence of the structure function is small (i.e. approximate scaling). The scattered quark has an invariant mass squared of order $Q^{2}$ and can radiate gluons. These in turn can produce quark-antiquark pairs or other gluons. This QCD shower process is thought to continue until the values of the momentum transfers involved are small (i.e. $\alpha_{\mathrm{s}}$ is large), at which point confined colourless clusters (hadrons) emerge. The hadronisation mechanism occurs on a time-scale much longer than that of the initial collision, and so it does not substantially affect the cross-section for the complete process.

\section{The Latest Surprise}

By about 1980 a muon beam, capable of producing muons up to $280 \mathrm{GeV}$ and $Q^{2}$ values up to about $100 \mathrm{GeV}^{2}$ was in operation at CERN. Muons are easier than electrons to handle experimentally. The European Muon Collaboration (EMC), a group comprised of members from 13 institutes, was in the process of taking data using various targets notably hydrogen and deuterium, so that the structure functions on both protons and neutrons could be measured. An iron target was also used, the main reason being to accumulate statistics more rapidly. Because the $Q^{2}$ values involved were large it was expected that nuclear effects were negligible, apart from the effects of the Fermi motion of the nucleons, for which a correction could be applied. Thus, apart from this correction and the small neutron to proton excess in iron, identical results for the structure function $F_{2}$ (per nucleon) to those measured on deuterium were expected. Hence it was yet another surprise in this subject when it was discovered that this was not the case. The ratio of $F_{2}$ on iron to deuterium was found to be above unity for small values of $x$ and below unity for large $x$, with a cross-over at about $x$ $\cong 0.2$. The degree of the surprise can be gauged by the fact that more than 100 theoretical papers, attempting to explain the effect, have been published since the discovery was announced in 1983.

Confirmation of the effect, which has come to be known as the EMC effect, soon came from experimenters at SLAC. Initially data, previously discarded, of interactions in the solid walls of targets were used, but later very precise measurements, for a whole series of nuclei, were carried out. Because of the kinematics of the set-up, the most precise data were at large $x$. The structure function ratio was found to decrease with increasing atomic mass, going from about 0.9 for $C / D$ to 0.8 for $A u / D$, both for $x \cong$

\section{Universität Bern}

Assistentin / Assistent

Physikalisches Institut Abt. Massenspektrometrie und Raumforschung

Wir suchen eine Experimentalphysikerin oder einen Experimentalphysiker mit Doktorat oder Diplom. Zum Aufgabenkreis gehören die Betreuung einer grossen Eichanlage für Weltraum lonenmassenspektrometer (für deren technischen Unterhalt und Betrieb ein Ingenieur besorgt ist), die Beteiligung am Unterricht (Praktika, Übungen) und die Mitarbeit an einem Forschungsprojekt aus den Gebieten Sonnenwind, Kometen, Magnetosphäre.

Erfahrung in den Gebieten Ionenoptik, Vakuumtechnik, Elektronik und Informatik ist wünschenswert aber nicht unbedingt Voraussetzung.

Die Dauer der Anstellung ist beschränkt.

Anmeldung an: Prof. H. Balsiger, Physikalisches Institut,

Uni Bern, Sidlerstr. 5, CH-3012 Bern, Tel. (31) 654411.

0.6 . A sharp rise in the ratio at very large $x(\gtrsim 0.8)$ was also observed, this being attributable to Fermi motion. At low $x$ the SLAC data showed no sizeable increase above unity. However, as the minimum $x$ value reached was greater than that where the rise above unity in the EMC data was observed, the results were not incompatible.

A second experiment, the Bologna CERN - Dortmund - Munich - Saclay (BCDMS) Collaboration, was also positioned in the muon beam-line at CERN. From measurements of the $F_{2}$ ratios on $\mathrm{N} / \mathrm{D}$ and $\mathrm{Fe} / \mathrm{D}$, a rise above unity at small $x$ was confirmed, but not as large as that suggested by the original EMC data. Further measurements were also carried out by the EMC using a set-up in which the potential systematic errors cancelled directly. The original EMC measurements were carried out at different times and with different configurations of the apparatus, so that the resulting systematic errors on the ratio were relatively large. Fig. 3 shows a comparison of the most accurate data sets produced for nuclei of medium $A$ values $(\mathrm{A}=\mathrm{Ca}, \mathrm{Fe}, \mathrm{Cu})$. The quantity plotted is $R_{\mathrm{EMC}}(x)=F^{\mathrm{A}}{ }_{2}(x) / F^{\mathrm{D}}{ }_{2}(x)$, that is, the ratio of the structure function per nucleon on the nuclear target compared to that for deuterium. The data are compatible with a small rise $\left(R_{\mathrm{EMC}} \cong 1.03\right)$ at $x \cong 0.15$. However, there is a turn-over at smaller $x$, with the ratio again falling below unity. This latter effect is seen in the new EMC data discussed above and confirmed by a dedicated study of the small $x$ and $Q^{2}$ region made with a special small angle trigger, also by the EMC. The data shown in Fig. 3 are averaged over all $Q^{2}$. Any $Q^{2}$ dependence is fairly small.

Thus a reasonably consistent experimental picture has become established. The quarks in the nuclear target carry a smaller momentum fraction (per nucleon) than those in deuterium at large $x$

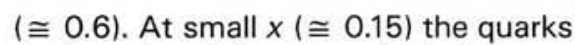
(and antiquarks) carry relatively more momentum in the nuclear target. The integrals of $F_{2}$, over the measured $x$ range, are close to zero, showing that there is a shift in the momentum distribution from high to low $x$ in the heavy target, with only a fairly small change in the gluon distribution.

The EMC effect has also been studied using incident neutrino beams. Because the weak interaction cross-section is small, the resulting event samples are somewhat smaller than those obtained with charged lepton beams. However the results are compatible.

\section{The Models}

Before considering the detailed models which have been proposed, it is worthwhile establishing the approximate distance scales involved. If $d_{R}$ is the inter-nucleon separation in the rest frame of the nucleus $\left(d_{R} \sim 1 / M_{\pi}\right.$, where $M_{\pi}$ is the pion mass), then in the Lorentz frame of the collision shown in Fig. 2 (the so-called Breit frame), this distance is Lorentz contracted to $d_{\mathrm{B}} \cong d_{\mathrm{R}} M_{\mathrm{N}} / P$, where $P$ is the nucleon momentum in this frame. From the Uncertainty Principle, the wavelength of the quark, antiquark or gluon (generically known as partons) is $\lambda \cong 1 /(x P)$, and hence this becomes comparable with the internucleon separation for a value of $x \cong$ $1 /\left(d_{\mathrm{R}} M_{\mathrm{N}}\right) \cong M_{\pi} M_{\mathrm{N}} \cong 0.15$. Although this argument is rather crude, it illustrates the point that partons at low $x(\leq 0.1)$ can no longer be considered as belonging to a specific nucleon. The deuteron is, however, loosely bound and, for the purpose of this discussion, can be considered as two (quasi) free nucleons.

Two broad classes of model have been developed to explain the region $0.1 \leq x$ $\lesssim 0.7$. The first category comprises 'conventional' nuclear physics models. In these models a summation is made of the contributions of all possible scatter- 
ing components of the nucleus. In addition to the traditional protons and neutrons, constituent pions and nucleon resonances (e.g. $\Delta$ ) have been considered. More exotic possibilities, such as confined 'bags' of $6,9,12$ etc. quarks existing within the nucleus, have also been proposed. There is sufficient flexibility in these models that most can be adjusted to give a plausible fit to the existing data. In the pion models, for example, the pion carriers of the nuclear force provide an additional target of quark-antiquark pairs for the virtual photon. Since the variable $x$ is measured as though the nucleon were the target, this pion contribution suffers a reduction by a kinematic factor $M_{\pi} / M_{N}$, and thus appears at small $x$. This model can thus explain the rise above unity of $R_{\mathrm{EMC}}$ at $x \cong 0.15$ and, by momentum conservation, the depletion at larger $x$. Crudely stated, an iron nucleus in the kinematic regime of the experiments consists of 26 protons, 30 neutrons and about 5 pions. Models containing constituents of the nucleus heavier than the nucleon also give a depletion at large $x$. A further, and related question is the role of Fermi motion and nuclear binding energy. However, models making a sophisticated treatment of binding energy do not predict a rise above unity at $x \cong 0.15$, and the data cannot be described by these effects alone.

In the above models it is assumed that the structure function of a particle in a nucleus is the same as the free particle structure function. That is, it is assumed that the internal QCD dynamics of the nuclear constituents are not influenced by the nuclear environment. $A$ different approach is taken in the second model, the so-called $Q C D$ rescaling model. Here it is assumed that the internal QCD dynamics are indeed modified in the nuclear environment, and that it is these changes which give rise to the observed effects. In $Q C D$, the $Q^{2}$ dependence of the structure functions, but not the functions themselves, can be predicted (in a perturbative expansion). The predicted pattern that, as $Q^{2}$ increases, there is a fall in the structure function at large $x$ and a rise at small $x$ has been verified for targets of different $A$ values. In the rescaling model, the evolution with $Q^{2}$ of $F_{2}^{A}\left(x, Q^{2}\right)$ is taken to be the same as $F_{2}^{D}\left(x, Q^{2}\right)$, but from a different starting point in $Q^{2}$. Thus the $Q^{2}$ value at which a nucleon in an iron nucleus has the same properties as a free nucleon is not the same (roughly $Q_{\mathrm{A}}^{2} \cong \xi\left(Q^{2}\right) Q_{\mathrm{D}}^{2}$, with $\xi \cong 2$ for $A=F e)$. Hence, for $a$ given $Q^{2}$, the heavy target structure functions are more evolved, so that the

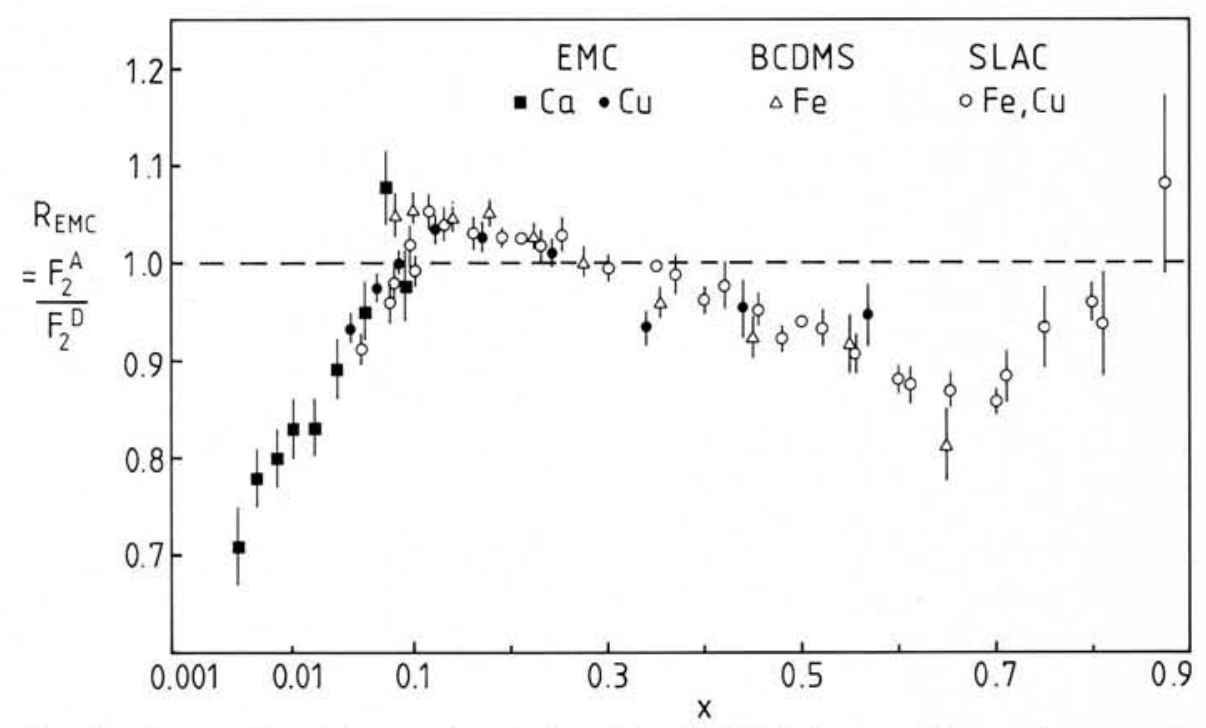

Fig. 3 - Compilation of data on the ratio $R_{E M C}(x)=F_{2}^{A}(x) / F_{2}(x)$ versus $x$ from various experiments. The scale in $x$ is logarithmic for $x<0.1$ and linear for $x \geq 0.1$.

ratio $F_{2}^{\mathrm{A}}\left(x, Q^{2}\right) / F_{2}^{\mathrm{D}}\left(x, Q^{2}\right)$ should follow the observed pattern of scaling violations. That is, $R_{\mathrm{EMC}}(x)$ should be less than unity at large $x$ and larger than unity at small $x$, with a cross-over at $x \cong 0.2$. From Fig. 3 it can be seen that this is the case; however, the precise cross-over point has not yet been established.

Although the QCD rescaling model uses $Q C D$, it is not required by $Q C D$. It has also been argued that the $Q C D$ rescaling model and the conventional nuclear physics approach could be alternative descriptions of the same underlying physics. If this is the case, a relationship exists between the QCD constants which dictate the $Q^{2}$ evolution and basic nuclear physics parameters (such as binding energies etc.). The predicted relationships are indeed roughly satisfied. All these models have limited ranges of applicability, however. The QCD model does not explain the rapid (Fermi motion) rise at large $x$, and none of these models predicted the turn-over for $x \geq 0.05$.

Studies with real photons have shown that the total cross-section per nucleon varies with $A$ as roughly $A^{2 / 3}$. These observations can be understood as follows. The incident photon undergoes quantum fluctuations to quark-antiquark pairs (this is required by $\mathrm{QED}$ ). These pairs have the same quantum numbers as the vector mesons $(\rho, \omega, \phi$, $\mathrm{J} / \mathrm{b}$ etc.), and it is assumed that they transform into virtual states of these particles. Since the mean free path of a strongly interacting particle in nuclear matter is small (typically a few fermis), the interaction with a nucleus is mainly a surface effect. Thus the other nucleons are in the shadow of the surface nucleons, and one expects $\sigma \sim \pi R^{2} \sim$
$A^{2 / 3}$. The shadowing process, via this vector meson dominance mechanism, may also be applicable to virtual photons in deep inelastic scattering. It is expected that this process is important at low $x(\leqq 0.05)$, and could explain the data shown in Fig. 3. However the model predicts that the effect dies out rapidly with increasing $Q^{2}$, and this dependence is excluded by the data. However the idea that, at low $x$, the quarks (and gluons) extend over more than one nucleon, and can recombine in such a way that their density is changed, can explain both the observed $x$ and $Q^{2}$ dependences.

\section{The Future}

Prior to the discovery of the EMC effect the structure of the nucleus was thought to be unimportant in the interpretation of deep inelastic scattering phenomena. This is no longer the case. This subject has proven to be a most fertile interdisciplinary area for both the nuclear structure and the elementary particle physics communities.

Further experimental investigation is in progress or is planned. Discrimination between the various models may be possible by a detailed study of the $Q^{2}$ dependence. Indeed, preliminary results suggest that many models will have difficulty in accommodating it. The ultimate goal is, however, an understanding of the nucleus in terms of QCD. This may well be some way off, but the existing and planned data on the EMC effect provide valuable clues in this quest.

\section{Further Reading}

A detailed review of the experiments and models can be found in Berger E.L. and Coester F., Annual Review of Nuclear Science 37 (1987) 463. 